\title{
COVERING LEMMAS REVISITED
}

\author{
by ANTHONY CARBERY
}

(Received 22nd July 1986)

\section{Introduction}

In this note we intend to discuss the method of A. Córdoba and R. Fefferman of using covering lemmas to control maximal functions, and make some simplifications which allow us to obtain alternative proofs of some of their results.

Let $\mathscr{B}$ be a collection of bounded open sets in $\mathbb{R}^{n}$, and let the associated maximal operator $M_{\mathscr{B}}$ be defined by $M_{\mathscr{B}} f(x)=\sup _{\mathscr{A} \ni R \ni x}(1 /|R|) \int_{R}|f|$ if $x \in \bigcup_{R \in \mathscr{B}} R$, and zero otherwise. Of interest to us will be the case when $\mathscr{B}$ is some collection of rectangles - the family of all cubes gives the Hardy-Littlewood maximal function, the family of all rectangles with sides parallel to the axes gives the strong maximal function. When $n \geqq 2$, we may let $\mathscr{B}$ be the collection of all rectangles pointing in one of a specified set of directions-and little is known about such maximal operations in general. The original impetus for this research was to try and open up to geometric methods the study of certain maximal functions which have hitherto only been treatable by the Fourier transform-notably when $\mathscr{B}$ is the family in $\mathbb{R}^{2}$ of rectangles with one side parallel to $\left(1,2^{j}\right)$, some $j \in \mathbb{Z}$. We have one small success with this maximal function-we are able to show geometrically that it is bounded in $L^{2}$; - see Section 3(c). Córdoba and Fefferman [4] had previously shown it to be weak type 2, and Nagel, Stein and Wainger [7] used the Fourier transform to obtain $L^{p}$ boundedness, $p>1$.

In a previous paper [1], S.-Y. A. Chang, J. Garnett and the author studied the problem of characterising the weights $\omega$ for which

$$
\int_{Q} M f \omega \leqq C \int_{Q}|f| \log ^{+}|f| \omega+C \omega(Q)
$$

holds whenever $f$ is supported in the cube $Q$ (where $M$ is the Hardy-Littlewood maximal function.) Using the methods of the present paper we are able to give a simplified (albeit still unsatisfactory) necessary and sufficient condition for (1) to hold. We do this in Section 3(d).

Some of the topics in this paper have also been treated in a similar but lengthier manner by Jawerth, [6].

\section{Covering lemmas}

One of the standard proofs of the Hardy-Littlewood maximal theorem reduces to the following covering property of cubes in $\mathbb{R}^{n}$ : there exists a constant $C(n)$ such that given any (finite) family of cubes in $\mathbb{R}^{n}$ we may extract a subfamily which is pairwise disjoint, 
and such that the measure of the union of the original family does not exceed $C(n)$ times the measure of the union of the selected subfamily. The method of Córdoba and Fefferman is to reduce an estimate of the form $M_{\infty}: L^{p} \rightarrow L^{p, \infty}(p>1)$ to an $L^{q}((1 / p)+$ $(1 / q)=1)$ analogue of the above property:

Theorem A ([3]). Let $1<p<\infty$. Then $M$ is of weak type $p$ if and only if $\mathscr{B}$ satisfies the property $V_{q}$ :

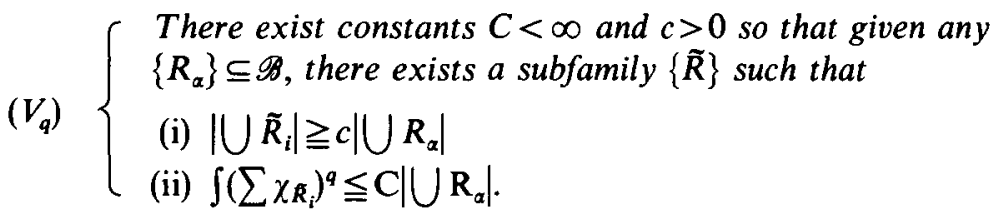

Notice that (as in the classical covering lemma for the Hardy-Littlewood maximal function), an element of strategy is needed to choose the subfamily satisfying (i) and (ii) when applying this theorem in any given situation. We shall show here that this element may be removed if the collection $\mathscr{B}$ of rectangles satisfies some mild extra requirement, such as, for example, being invariant under the usual isotropic dilations of $\mathbb{R}^{n}$.

Let us fix some notation. For $\alpha \in \mathbb{Z}^{n}$ let $Q_{\alpha}$ be the unit cube centred at $\alpha ; A$ will denote any finite subset of $\mathbb{Z}^{n}$ with cardinality $\# A . \mathscr{B}_{1}$ will denote the subfamily of $\mathscr{B}$ consisting of members of $\mathscr{B}$ all of whose sides are greater than or equal to one. For $\alpha \in \mathbb{Z}^{n}, R_{\alpha}$ will denote some rectangle in $\mathscr{B}_{1}$ with $R_{\alpha} \cap Q_{\alpha} \neq \varnothing$. Finally, $(1 / p)+(1 / q)=1$.

Theorem 1. Let $1<p<\infty$, and let $\mathscr{B}$ be a collection of rectangles in $\mathbb{R}^{n}$ invariant under dilations. Then $M_{\mathscr{B}}$ is of weak type $p$ if and only if there is a constant $C_{q}$ such that whenever $R_{\alpha} \in \mathscr{B}_{1}, R_{\alpha} \cap Q_{\alpha} \neq \varnothing$, and $A \subseteq \mathbb{Z}^{n}$, then

$$
\int\left(\sum_{\alpha \in A} \frac{\chi_{R_{\alpha}}}{\left|R_{\alpha}\right|}\right)^{q} \leqq C_{q}(\# A) .
$$

Theorem 2. Let $1<p<\infty$, and $\mathscr{B}$ be as above. Then $M_{\mathscr{D}}$ is of strong type $p$ if and only if there exists a constant $C_{q}$ such that whenever $R_{a} \in \mathscr{B}_{1}, R_{\alpha} \cap Q_{\alpha} \neq \varnothing$, then

$$
\int\left(\sum \lambda_{\alpha} \frac{\chi_{R_{\alpha}}}{\left|R_{\alpha}\right|}\right)^{q} \leqq C_{q} \sum\left|\lambda_{\alpha}\right|^{q} .
$$

Remarks. 1. Theorems 1 and 2 are, in fact, very natural corollaries of the method used by Córdoba [2] to control the maximal function associated to rectangles of a given eccentricity in $\mathbb{R}^{2}$, and seem more straightforward to apply than Theorem $A$. It therefore seems surprising that Córdoba and Fefferman did not employ Theorem 2 in some of their work.

2. The hypothesis that $\mathscr{B}$ consist of rectangles and be invariant under dilations may be relaxed. We leave it to the reader to formulate the more general theorems.

3. Variants of Theorems 1 and 2 will hold if we are studying the behaviour of $M$ from one Banach space to another; all that is required is that the function spaces have 
suitable duals. This observation allows us to characterise the weights $\omega$ for which (1) holds. See Section 3(d).

4. Consequently there is no "weak type 1" theory, as in the case of Theorem A. However, while $V_{\infty}$ holds, for example, when $\mathscr{B}$ is the family of cubes, the analogue of (2) is false for cubes. In general, one seems to gain an extra constant $1 /(p-1)$ when applying Theorem 1: put another way, a shortcoming of the method is that if a maximal function actually is bounded from $L(\log L)^{k}$ to weak $L^{1}$, the best one can hope to prove using Theorem 1 is that $L(\log L)^{k+1}$ goes to weak $L^{1}$.

The following two lemmas are elementary:

Lemma 1. Let $\mathscr{B}_{2}$ consist of all rectangles in $\mathscr{B}_{1}$ expanded by a factor $2 \sqrt{n}+1$. Then there exists a constant $C_{n}$ such that

$$
M_{\mathscr{P}_{1}} f(x) \leqq \sum_{\alpha \in Z^{n}} \sup _{R_{\alpha}} \frac{1}{\left|R_{\alpha}\right|} \int_{R_{\alpha}}|f| \cdot \chi_{Q_{\alpha}}(x) \leqq C_{n} M_{\mathscr{O}_{2}} f(x) .
$$

Lemma 2. Let $\mathscr{B}$ be invariant under dilations. Then $M_{\mathscr{B}}$ is strong (weak) type $p \Leftrightarrow M_{\mathscr{B}_{1}}$ is strong (weak) type $p$.

Thus in proving theorems one and two we may replace $M f$ by $T f(x)=$ $\sum_{\alpha \in Z^{n}}\left(1 /\left|R_{\alpha}\right|\right) \int_{R_{\alpha}} f \chi_{Q_{\alpha}}(x)$ and obtain constants independent of the choice of $\left\{R_{\alpha}\right\} \subseteq \mathscr{B}_{1}$, $R_{a} \cap Q_{\alpha} \neq \varnothing$.

Proofs of Theorems 1 and 2. If $M$ is strong (weak) type $p$, then $T^{*}$ defined by $T^{*} h(x)=$ $\sum_{\alpha} \int_{Q_{\alpha}} h\left(\chi_{R_{\alpha}} / R_{\alpha} \mid\right)(x)$ is bounded from $L^{q}\left(L^{q, 1}\right)$ to $L^{q}$. Setting $h=\sum \lambda_{\alpha} \chi_{Q_{\alpha}}\left(\chi_{\cup_{\alpha \in A} Q_{\alpha}}\right)$ yields (3) ((2)). If now $\mathscr{B}$ satisfies (3), $T^{*}$ satisfies

$$
\int\left(T^{*} h\right)^{q} \leqq C_{q} \sum_{\alpha}\left(\int_{Q_{A}} h\right)^{q} \leqq C_{q} \int|h|^{q}
$$

and therefore $T$ and $M$ are of strong type $p$. Finally, if $\mathscr{B}$ satisfies (2), $\lambda>0$ and $A=\left\{\alpha \mid\left(1 /\left|R_{\alpha}\right|\right) \int_{R_{\alpha}} f>\lambda\right\}$, then

$$
\begin{aligned}
\{x \mid T f(x)>\lambda\} & =\left|\bigcup\left\{Q_{\alpha} \mid \frac{1}{\left|R_{\alpha}\right|} \int_{R_{\alpha}} f>\lambda\right\}\right| \\
& =\# A \\
& \leqq \frac{1}{\lambda} \sum_{\alpha \in A} \frac{1}{\left|R_{\alpha}\right|} \int_{R_{\alpha}} f \\
& \leqq \frac{1}{\lambda}\|f\|_{p}\left\|\sum_{\alpha \in A} \frac{\chi_{R_{A}}}{\left|R_{\alpha}\right|}\right\|_{q} .
\end{aligned}
$$

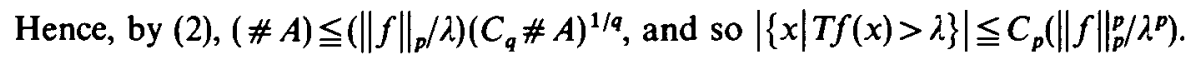




\section{Applications}

(a) The family of cubes in $\mathbb{R}^{n}$. We shall use Theorem 1 to show the weak type $p, p>1$ of the Hardy-Littlewood maximal operator. (It is interesting to notice that if we try and apply Theorem 2 we would first require the strong type for the analogue of the maximal operator for sequences, cf. Hardy and Littlewood [5].) We partially order the family of cubes $\mathscr{B}$ by $R \leqq S$ if $R \cap S \neq \varnothing$ and $|R| \leqq|S|$. Observe that with this definition, $\#\left\{\alpha \mid R_{\alpha} \leqq R\right\} \leqq C|R|$ (where $R_{\alpha}, R \in \mathscr{B}_{1}, R_{\alpha} \cap Q_{\alpha} \neq \varnothing$ ). Thus if $q \in \mathbb{N}$,

$$
\int\left(\sum_{\alpha \in A} \frac{\chi_{R_{\alpha}}}{\left|R_{\alpha}\right|}\right)^{q} \leqq C q ! \sum_{\alpha: R_{\alpha_{1}} \leqq R_{\alpha_{2}} \leqq \cdots \leqq R_{\alpha_{q}}} \frac{\left|R_{\alpha_{1}}\right|}{\left|R_{\alpha_{1}}\right| \ldots\left|R_{\alpha_{q}}\right|} \leqq q ! C^{q} \# A .
$$

(b) The family of rectangles in $\mathbb{R}^{n}$ with sides parallel to the axes. For simplicity, we shall deal only with the case $n=2$, using the Hardy-Littlewood maximal function to control the strong maximal function. (In $\mathbb{R}^{n}$, one proceeds inductively, dealing successively with rectangles $(n-k)$ of whose sides are equal, for $k=0,1, \ldots, n-1$.) We shall obtain (3) from the corresponding estimate for cubes when $q \in \mathbb{N}$, and we shall assume that our rectangles are dyadic, and have long side pointing in the $x_{1}$-direction. Order such rectangles by $R \leqq T$ if $R \cap T \neq \varnothing$, and the long side of $R$ is less than or equal to the long side of $T$. If now $R_{\alpha} \leqq R, R_{\alpha}, R \in \mathscr{B}_{1}, R_{\alpha} \cap Q_{\alpha} \neq \varnothing$, and $S_{\alpha}$ is the dyadic cube containing $Q_{\alpha}$ of side the small side of $R_{\alpha}$, then $\left|R_{\alpha} \cap R\right| /\left|R_{\alpha}\right|=\left|S_{\alpha} \cap R\right| /\left|S_{\alpha}\right|$; if $R_{\alpha_{1}} \leqq R_{\alpha_{2}}, \ldots, R_{\alpha_{q}}$, then $R_{\alpha_{1}} \leqq R_{\alpha_{2}} \cap \cdots \cap R_{\alpha_{q}}$ and so

$$
\frac{\left|R_{\alpha_{1}} \cap \cdots \cap R_{\alpha_{q}}\right|}{\left|R_{\alpha_{1}}\right|}=\frac{\left|S_{\alpha_{1}} \cap R_{\alpha_{2}} \cap \cdots \cap R_{\alpha_{q}}\right|}{\left|S_{\alpha_{1}}\right|}
$$

Therefore,

$$
\begin{aligned}
\int\left(\sum \lambda_{\alpha} \frac{\chi_{R_{\alpha}}}{\left|R_{\alpha}\right|}\right)^{q} & \leqq C_{q} \sum_{R_{\alpha_{1}} \leqq R_{\alpha_{2}} \cdots \cdots R_{\alpha}} \lambda_{\alpha_{1}} \ldots \lambda_{\alpha_{q}} \frac{\left|R_{\alpha_{1}} \cap \cdots \cap R_{\alpha_{q}}\right|}{\left|R_{\alpha_{1}}\right| \cdots\left|R_{\alpha_{q}}\right|} \\
& =C_{q} \int\left(\sum_{\alpha} \lambda_{\alpha} \frac{\chi_{S_{\alpha}}}{\left|S_{\alpha}\right|}\right)\left(\sum_{\beta} \lambda_{\beta} \frac{\chi_{R_{\beta}}}{\left|R_{\beta}\right|}\right)^{q-1} .
\end{aligned}
$$

So by Hölder's inequality,

$$
\begin{aligned}
\int\left(\sum \gamma_{\alpha} \frac{\chi_{R_{A}}}{\left|R_{\alpha}\right|}\right)^{q} & \leqq\left(C_{q}\right)^{q} \int\left(\sum \lambda_{\alpha} \frac{\chi_{s_{\alpha}}}{\left|S_{\alpha}\right|}\right)^{q} \\
& \leqq C_{q} \sum\left|\lambda_{\alpha}\right|^{q}
\end{aligned}
$$

since $\left\{S_{\alpha}\right\}$ is a family of cubes for which (3) holds.

Notice that (2) for rectangles in the case $q=2$ implies that whenever $\left\{R_{a}\right\} \subseteq \mathscr{B}_{1}$, $R_{\alpha} \cap Q_{\alpha} \neq \varnothing$, then there is at least one $R_{\alpha_{0}}$ for which $\sum_{\alpha}\left|R_{\alpha} \cap R_{\alpha_{0}}\right| /\left|R_{\alpha}\right| \leqq C\left|R_{\alpha_{0}}\right|$. However, there does not seem to be a simple geometric criterion for determining which rectangle should be $R_{\alpha_{0}}$. This phenomenon may still occur when $\mathscr{B}$ is a one-parameter family of rectangles. 
(c) The family of rectangles in lacunary directions in $\mathbb{R}^{2}$. To see that the maximal operator here is strong type 2, order the rectangles by longest sidelength; Córdoba and Fefferman [4] observed that if $R_{\alpha} \leqq R$, then $\left|R_{\alpha} \cap R\right| /\left|R_{\alpha}\right| \leqq\left|S_{\alpha} \cap R\right| /\left|S_{\alpha}\right|$ where $S_{\alpha}$ is the triple of the smallest rectangle with sides parallel to the axes which contains $R_{\alpha}$. Arguing as in (b) and using the result from (b) concludes the proof.

(d) Weighted inequalities for the Hardy-Littlewood maximal function near $L^{1}$. In [1], Chang, Garnett and the author gave a necessary and sufficient condition on a nonnegative locally integrable weight $\omega$ that

$$
\int_{\mathbf{Q}} M f \omega \leqq C \int_{\mathbf{Q}}|f| \log ^{+}|f| \omega+c \omega(Q)
$$

hold whenever $f$ is supported in the cube $Q, M$ denoting the Hardy-Littlewood maximal operator. This condition was very ugly, and really amounted to saying that $\omega$ satisfied a "strong-type exponential" covering lemma involving doubly-indexed families of cubes. (See also Jawerth [6].) Assuming without loss of generality that $M$ and $Q$ are dyadic, and using Theorem 2 and Remarks 2 and 3 following its statement, we come immediately to:

Theorem 3. The weight $\omega(x)$ satisfies (1) if and only if

$$
\sup _{Q} \sup \int_{Q} \exp \left\{\varepsilon \sum_{\alpha: T_{\alpha} \subseteq Q} \frac{\chi_{R_{\alpha}}(x) \omega\left(T_{\alpha}\right)}{\omega(x)\left|R_{\alpha}\right|}\right\} \frac{\omega d x}{\omega(Q)}<\infty
$$

for some $\varepsilon>0$, the inner sup being taken over all decompositions of $Q$ into congruent dyadic subcubes $T_{\alpha}$, and over all choices $R_{\alpha}$ of a dyadic cube with $T_{\alpha} \subseteq R_{\alpha} \subseteq Q$.

It is tempting to speculate that perhaps the 'canonical' choice $R_{\alpha}=T_{\alpha}$ in (4) may give a sufficient condition for (1) to hold. Unfortunately this is not the case, since (4) with the choice $R_{\alpha}=T_{\alpha}$ is certainly implied by

$$
\sup _{Q} \int_{Q} \exp \left\{\varepsilon^{\prime} \frac{M\left(\omega \chi_{Q}\right)}{\omega}\right\} \frac{\omega d x}{\omega(Q)}<\infty
$$

which is equivalent to

$$
\sup _{Q} \int_{Q} \exp \left\{\varepsilon^{\prime \prime} \frac{\omega_{Q}}{\omega}\right\} \frac{\omega d x}{\omega(Q)}<\infty,
$$

and (6) in [1] was shown to be insufficient for (1) to hold.

Lemma 3. (5) and (6) are equivalent.

Proof. Clearly (5) implies (6), so assume (6) holds. Let $A_{p}(\omega)=$ $\sup _{Q}\left\{\int_{Q}\left(\omega_{Q} / \omega\right)^{q}(\omega d t / \omega(Q))\right\}^{p-1}$ denote the $A_{p}$ constant of $\omega$. It is shown in [1] that (6) 
implies $A_{p}(\omega)=0(1 / p-1)$ as $p \downarrow 1$. A careful perusal of the paper of Sawyer [8] reveals that

$$
\int|M f|^{p} \sigma \leqq C^{p+q} A_{p}^{q}(\sigma) p q \int|f|^{p} \sigma, \quad p>1 .
$$

Therefore

$$
\begin{aligned}
\int_{Q} \exp \left\{\varepsilon^{\prime} \frac{M\left(\omega \chi_{Q}\right)}{\omega}\right\} \frac{\omega d x}{\omega(Q)} & \left.=\sum_{k=0}^{\infty} \frac{\varepsilon^{\prime k}}{k !} \int_{Q}\left(\frac{M\left(\omega \chi_{Q}\right)}{\omega}\right)^{k} \frac{\omega d x}{\omega(Q)}\right) \\
& \leqq C+\sum_{k=2}^{\infty} \frac{\varepsilon^{\prime k}}{k !} C^{k} A_{k}^{k^{\prime}}\left(\omega^{-1 /(k-1)}\right) k k^{\prime} \int_{Q} \frac{\omega d x}{\omega(Q)} \\
& \leqq C+C^{\prime} \sum_{k=2}^{\infty} \frac{\left(C \varepsilon^{\prime}\right)^{k}}{k !} A_{k^{\prime}}^{k^{\prime}(k-1)}(\omega) k k^{\prime} \\
& \leqq C+C^{\prime} \sum_{k=z}^{\infty} \frac{\left(C \varepsilon^{\prime}\right)^{k}}{k !} k^{k+1} \leqq C^{\prime \prime}
\end{aligned}
$$

if $\varepsilon^{\prime}$ is sufficiently small.

\section{REFERENCES}

1. A. Carbery, S.-Y. A. Chang and J. Garnett, Weights and $L \log L$, Pacific J. Math. 120 (1985), 33-45.

2. A. CовдовA, The Kakeya maximal function and the spherical summation multipliers, Amer. J. Math. 99 (1977), 1-22.

3. A. Córdoba and R. Fefferman, A geometric proof of the strong maximal theorem, Ann. of Math. 102 (1975), 95-100.

4. A. Cordoba and R. Fefferman, On differentiation of integrals, Proc. Nat. Acad. Sci. U.S.A. 74 (1977), 2211-2213.

5. G. H. Hardy and J. E. Litrlewood, A maximal theorem with function-theoretic applications, Acta Math. 54 (1930), 81-116.

6. B. JAWERTH, Weighted inequalities for maximal operators: Linearization, localization factorization, Amer. J. Math., 108 (1986), 361-414.

7. A. Nagel, E. M. Stein and S. Wainger, Differentiation in lacunary directions, Proc. Nat. Acad., Sci. U.S.A. 75 (1978), 1060-1062.

8. E. SAWYER, Two Weight Norm Inequalities for certain Maximal and Integral Operators (Springer Lecture Notes in Math. 908 1982), 102-127.

Mathematics Division

UNIVERSITY OF SUSSEX

FALMER

Brighton BN1 9QH 